CHAPITRE 2

\title{
La fusion par confinement inertiel
}

MICHEL ANDRÉ, CHARLES BAYER, MICHEL DECROISETTE, CLAUDE DEUTSCH, DENIS JURASZEK, BRUNO LE GARREC ET ARNOLD MIGUS 



\section{Principes et motivations}

\subsection{Concept}

L'idée à la base de la fusion par confinement inertiel (FCI) est de faire imploser une capsule sphérique (également appelée cible) contenant un mélange de deutérium et de tritium.

Pour réaliser le processus, la cible est attaquée par un rayonnement très intense ; la paroi externe, transformée en plasma sous l'effet de l'élévation de température, se détend vers le vide ce qui, par effet fusée, entraîne l'implosion de la capsule et la compression du mélange fusible. Sous certaines conditions, par l'effet de la coalescence d'ondes de choc, la densité et la température atteignent au cœur de la cible comprimée les valeurs requises pour que s'instaurent les réactions de fusion. Tant que cette situation se maintient, $c^{\prime}$ est-à-dire avant que la cible se désagrège, l'énergie thermonucléaire se dégage ( $d$ 'où le terme de confinement inertiel).

Les travaux théoriques réalisés depuis les années soixante ont permis de dégager plusieurs lois essentielles qu'il faut respecter pour réaliser la fusion inertielle. On peut retenir en particulier que :

- l'énergie à investir dans le milieu fusible pour obtenir l'allumage (situation obtenue lorsque le bilan énergétique du milieu devient positif) varie comme l'inverse du carré de sa densité. Il est donc souhaitable d'atteindre des densités élevées ( $c$ 'est-à-dire comprimer fortement le milieu fusible) pour minimiser cette énergie primaire ;

- pour obtenir des gains élevés (rapport de l'énergie thermonucléaire dégagée à l'énergie initialement délivrée à la cible), il faut ne porter aux conditions d'allumage qu'une partie du combustible. C'est le concept d'allumage par point chaud central.

\subsection{Principe d'un réacteur $\mathrm{FCl}$}

Un réacteur à fusion par confinement inertiel comporte quatre parties principales. 


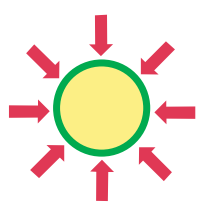

Chauffage de la cible par la source d'énergie

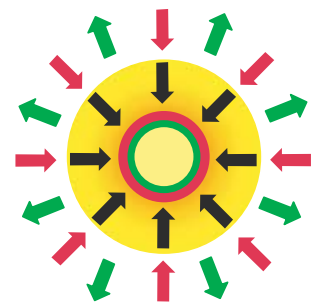

Création d'un plasma qui comprime le DT

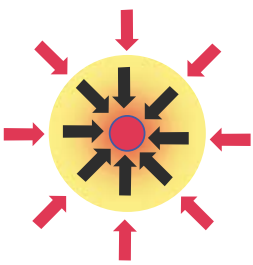

Ignition à partir d'un point chaud

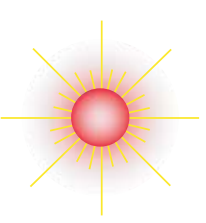

Combustion

Figure 2.1

Phénoménologie de l'implosion.

Le driver

Alimenté par son banc d'énergie, il est chargé d'apporter l'énergie requise pour imploser la cible. Dans le cas d'un réacteur, il doit présenter les caractéristiques suivantes :

- énergie : qq. MJ (millions de joules); Intensité sur cible : qq. $10^{14} \mathrm{~W} / \mathrm{cm}^{-2}$; durée : qq. $10 \mathrm{~ns}$ (milliardièmes de seconde).

L'énergie doit être délivrée avec des contraintes particulières : focalisation sur une surface de dimension caractéristique inférieure ou égale à celle de la cible, loi de puissance adaptée à la dynamique de l'implosion, uniformité d'éclairement de la cible, équilibrage des puissances entre faisceaux, ...

- rendement $\geq 10 \%$;

- fonctionnement reproductible avec une fréquence de répétition de quelques hertz.

Parmi les sources présentant ces potentialités, sont en course aujourd'hui les accélérateurs d'ions lourds, les lasers de puissance à verre dopé au $\mathrm{Nd}$ et les strictions magnétiques.

La chambre de réactions

En son centre ont lieu les micro-explosions produisant l'énergie de fusion thermonucléaire. Elle assure une quadruple fonction : 
- elle permet de maintenir un vide suffisant pour que l'énergie du driver puisse se propager jusqu'à la cible ;

- elle constitue la première barrière de confinement entre le foyer thermonucléaire que l'on peut considérer comme ponctuel, et l'environnement;

- elle récupère sous forme thermique l'énergie dégagée, en ralentissant et absorbant particules et photons dans une « couverture »; un fluide caloporteur véhicule cette énergie à l'extérieur;

- elle est le siège des réactions secondaires qui régénèrent le tritium nécessaire au fonctionnement du réacteur.

On peut noter ici la similitude de certaines problématiques avec le confinement magnétique.

L'unité de traitement des matériaux et la centrale de production des cibles

La gestion des matériaux intervenant dans le fonctionnement de la chambre concerne à la fois les dégagements gazeux des cibles, la couverture et les matériaux de protection des parois.

Les générateurs de vapeur et les turbines

Ils transforment l'énergie thermique apportée par des fluides caloporteurs en énergie mécanique, puis en énergie électrique pour alimenter en électricité le réseau de distribution.

Pour fixer les idées, admettons qu'avec une énergie primaire de $10 \mathrm{MJ}$ une cible ait un gain de 100, un réacteur pourrait fournir une puissance électrique de $1500 \mathrm{MW}$ en fonctionnant à une fréquence de répétition de $7 \mathrm{~Hz}$.

\subsection{Les attraits de la fusion inertielle}

À la suite de la description sommaire précédente, on peut émettre quelques commentaires sur l'intérêt d'un réacteur à fusion inertielle.

Le plus remarquable est qu'au plan de la sûreté, comme pour un réacteur à fusion magnétique, il n'y a aucun risque d'emballement, car en cas d'incident, I'interruption de l'alimentation en cibles ou l'arrêt du driver stoppe le réacteur; $\mathrm{d}^{\prime}$ autre part, le fonctionnement $\mathrm{n}^{\prime}$ induit pas de transports de matières radioactives (déchets en particuliers). 
La construction modulaire présente l'avantage de limiter à la chambre et à I'unité de traitement des matériaux et de fabrication cibles, la présence de tritium et de matériaux activés. C'est une facilité pour la maintenance du driver ou même sa modification pour tenir compte d'avancées technologiques.

La quantité de matière nucléaire présente dans le réacteur est faible : il s'agit du tritium localisé essentiellement dans la couverture et dans la centrale de production des cibles; un développement technologique approprié (par exemple sur la fabrication des cibles cryogéniques) devrait conduire à un inventaire très réduit.

La puissance délivrée peut être adaptée très souplement en faisant varier la cadence des tirs sans affecter le rendement de fusion.

\subsection{Les principales étapes de la fusion inertielle}

Une stratégie de développement peut être envisagée pour démontrer progressivement que la fusion inertielle peut être une source d'énergie sure, propre, abondante et économique. II pourrait se dérouler en quatre étapes, à partir de la situation actuelle :

- démonstration de l'allumage (cette phase est en cours);

- démonstration d'un gain élevé ;

- démonstration industrielle;

- démonstration commerciale.

Si l'on se reporte à la description de la structure de principe, on remarque que la réalisation des quatre parties principales d'un réacteur (indépendantes et caractérisées par des technologies différentes) pourrait s'envisager en parallèle, ce qui serait sans doute un facteur de commodité et d'économie.

\section{Solutions et projets}

\subsection{Les drivers}

Parmi les sources présentant les potentialités requises, sont en course aujourd'hui les lasers de puissance, les accélérateurs d'ions et les strictions magnétiques. 
Les lasers de puissance

Le laser impulsionnel se présente comme un excellent candidat pour les premières étapes du développement de la $\mathrm{FCl}$ : grâce à ses propriétés - cohérence et monochromaticité - il est possible de focaliser son rayonnement dans un volume très petit et $d$ 'obtenir les densités de puissance requises pour réaliser en laboratoire des expériences représentatives même avec des énergies « modestes ».

Divers types de lasers sont envisageables, mais il semble acquis que la démonstration de faisabilité de la fusion inertielle sera réalisée avec un laser à verre dopé au néodymium. Sa technologie a en effet bénéficié de développements très poussés depuis la fin des années soixante, et elle est retenue pour les deux plus grandes installations en cours de construction : le LMJ et le NIF (voir § 3). En particulier, les problèmes posés par l'interaction laser-plasma sont traités dans le chapitre $4 \$ 1.1$.

\section{Les accélérateurs d'ions}

Les difficultés inhérentes aux lasers actuels : faible rendement, faible cadence à très forte énergie ainsi que les difficultés pour trouver des solutions sûres pour la tenue des dernières optiques dans le cas d'un réacteur, ont conduit à considérer d'autres techniques, en particulier les faisceaux de particules.

L'idée est de focaliser l'énergie sur la cible sous forme de faisceaux intenses d'ions énergétiques au lieu de rayonnement lumineux. Les accélérateurs de particules ont des arguments favorables au regard de la $\mathrm{FCl}$ : fiabilité, grande durée de vie, taux de répétition élevé (quelques dizaines de $\mathrm{Hz}$ ), rendement important (20 à $30 \%)$.

Divers types d'accélérateurs ont été étudiés, mais il est généralement admis que lorsque les lasers auront démontré la possibilité de brûler du DT en laboratoire et $d^{\prime}$ atteindre des gains de cibles de $\sim 10$, les ions lourds pourraient être le vecteur le mieux adapté pour les applications énergétiques.

Les projets de ce type de machine sont nombreux, mais aucun ne prétend aboutir à la démonstration de l'ignition à court terme.

\section{Les strictions magnétiques}

Dans une striction magnétique (ou Z-Pinch), on exploite la force de Laplace liée au passage d'un courant intense pour comprimer un "liner » (réseau de 
fils métalliques). La conversion de l'énergie cinétique en énergie interne produit un fort dégagement de rayonnement $X$. Ce rayonnement peut être utilisé pour imploser une cible.

\subsection{La cible et les schémas d'implosion}

Dans son principe, la cible est constituée d'une coquille en matériau léger sur la paroi interne de laquelle est déposé le combustible (DT) sous forme d'une couche cryogénique. La structure de cette cible doit répondre à des contraintes sévères : la sphéricité et l'uniformité d'épaisseur de la couche de DT doivent être respectées à environ $1 \%$, et la rugosité doit être inférieure au micromètre.

Diverses approches ont été envisagées pour réaliser l'implosion de la coquille.

\section{L'attaque directe}

Ce schéma se rapporte essentiellement à la situation d'une cible irradiée directement par les faisceaux d'un laser, comme le montre la figure. L'interaction à haute intensité entre le rayonnement laser et la paroi de la cible crée un plasma très chaud qui se détend; cette détente génère une pression dite " $d$ 'ablation " très élevée, qui provoque l'implosion de la cible.

La question est d'assurer une irradiation du microballon la plus uniforme possible pour préserver la structure sphérique de l'implosion, en minimisant les défauts d'éclairement liés au recouvrement par un nombre fini de faisceaux (défauts de pointage et de synchronisme, déséquilibre en énergie et en puissance), ainsi que les défauts propres de chaque faisceau (liés à la cohérence du rayonnement) qui sont des germes pour les instabilités hydrodynamiques.

\section{L'attaque indirecte}

Dans le cas de l'interaction entre le rayonnement laser et un milieu de numéro atomique élevé comme l'or, le plasma émet un rayonnement $X$ intense. Ceci a conduit à concevoir une approche "indirecte »: le rayonnement laser est absorbé dans une enceinte (ou " hohlraum ») renfermant la cible, et converti (par une succession de processus élémentaires absorption-chauffage-réémission) en un rayonnement $X$ qui réalise l'implosion. La cible est donc immergée dans un "four ». Au plan de l'uniformité de l'éclairement, les contraintes sur la quantité et la qualité des faisceaux laser sont notablement réduites par rapport à l'attaque directe. 
Le schéma d'attaque indirecte peut être aisément adapté au cas de faisceaux d'ions : les faisceaux irradient des convertisseurs (matériau de numéro atomique faible dopé d'un matériau de numéro atomique élevé, faisant office de " radiateurs » de rayonnement $\mathrm{X}$ ) situés à l'entrée de la cavité.

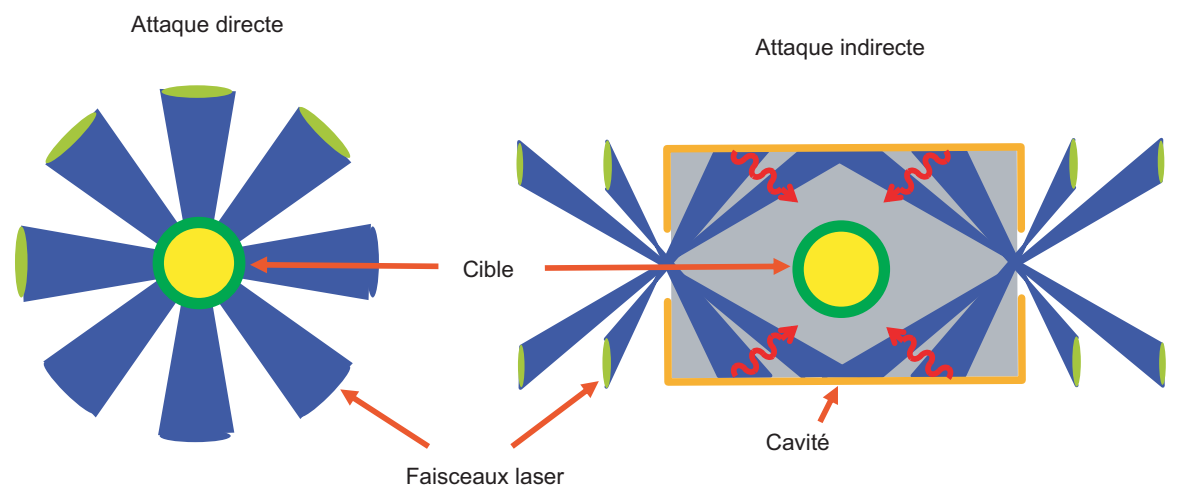

Figure $\mathbf{2 . 2}$

Schémas d'attaques directe et indirecte.

L'allumage rapide

Comme nous l'avons dit précédemment, la recherche d'un gain élevé conduit à mettre en œuvre un allumage par " point chaud " central, consistant à ne porter aux conditions d'allumage que le cœur du DT comprimé.

Un autre concept a été proposé, appelé allumage " rapide " ou allumage " latéral » qui consiste à dissocier les phases de compression et d'allumage : on comprime à des densités élevées l'ensemble du combustible, puis on chauffe sélectivement une petite fraction du DT pour le porter aux conditions d'allumage, à l'aide d'un rayonnement laser auxiliaire attaquant latéralement le combustible comprimé. Cette méthode est basée sur l'emploi de lasers à impulsion courte et ultra-haute intensité dont la technologie s'est développée au cours de la dernière décennie.

\subsection{La chambre de réactions et les projets de réacteurs}

On trouve dans la littérature de nombreux concepts de réacteurs publiés dans les trente dernières années, essentiellement aux États-Unis et au Japon, et peu en Europe (aucun projet français). 
Pour la chambre, le problème le plus crucial est celui de la première paroi matérielle, en vue directe de la micro-explosion, qui risque de se vaporiser ou se sublimer sous l'effet du dégagement d'énergie. Plusieurs solutions ont été explorées, mais l'idée d'un "mur sacrificiel » qui se régénère après chaque explosion est souvent retenue. On note par exemple la proposition d'un mur " movillé » en surface par l'écoulement gravitaire à travers une paroi poreuse d'un liquide de protection, ou bien d'une paroi épaisse constituée d'un fluide circulant à grande vitesse et absorbant la majeure partie de l'énergie de fusion.

L'analyse des projets de réacteurs montre que plusieurs options de chambre apparaissent viables, et que chaque type de driver peut être adapté à diverses solutions. Toutefois, la tendance suivante se dégage :

- comme les faisceaux laser peuvent être focalisés avec précision à longue distance et que la protection des optiques implique un éloignement de la cible important, les concepts de chambre pour des drivers laser se sont plutôt orientés vers les solutions « mur sec »;

- pour les drivers à ions lourds, on cherche à réduire la distance à la cible, et la solution d'un « mur liquide épais » est préférée.

Remarquons que, malgré la modularité de structure d'un réacteur inertiel, les trois composantes driver, cible, chambre, déterminent des interfaces posant des problèmes importants, comme la focalisation ou l'injection des cibles.

\section{3 | Situation actuelle et perspectives}

À ce jour, aucune démonstration de la combustion d'un mélange DT à l'aide $d^{\prime}$ 'un driver de laboratoire $n^{\prime}$ a encore été réalisée ; mais cette première phase du développement de la fusion inertielle est en cours, comme nous le verrons plus loin. Notons toutefois qu'aux États-Unis, des informations essentielles ont été obtenues grâce à des expériences baptisées "Centurion-Halite 》 $(\mathrm{H} / \mathrm{C})$, exécutées lors de tirs souterrains réalisés sur le site du Nevada. Ces expériences consistaient à imploser des cibles de type $\mathrm{FCl}$ en utilisant l'énergie délivrée par un engin nucléaire. Effectuées avec une puissance primaire élevée, elles ont apporté des précisions sur les conditions nécessaires à l'allumage et à la combustion du DT ; elles ont permis aux États-Unis d'asseoir la définition du laser NIF destiné à obtenir l'allumage en laboratoire (voir également plus loin).

En revanche, une somme importante de connaissances a été recueillie par la communauté scientifique, sur la physique de l'interaction rayonnement-matière 
(c'est-à-dire l'irradiation de la cible par l'énergie primaire du driver) ainsi que sur l'implosion d'une cible.

\subsection{L'implosion par laser}

La physique de l'interaction laser-plasma est étudiée depuis quarante ans et I'on en a une connaissance très approfondie - même si elle est encore incomplète - grâce aux multiples travaux réalisés un peu partout dans le monde let en majorité avec des lasers à verre au Néodyme). On sait en particulier parfaitement définir les conditions de longueur d'onde laser, d'intensité, de nature de cible qui optimisent le dépôt d'énergie.

Des études sur l'implosion par laser sont poursuivies par tous les grands laboratoires; notons en particulier : le CEA en France, LLNL et LLE aux États-Unis, ILE au Japon, Arzamas-16 en Russie. Ces travaux s'attachent en particulier à comprendre et maîtriser les phénomènes limitatifs, principalement le développement d'instabilités hydrodynamiques.

En analysant en termes de performances d'implosion les résultats obtenus avec les plus grandes installations, on peut dire que les valeurs des paramètres essentiels (symétrie d'irradiation, rapport de convergence de l'implosion (rapport du rayon initial de la coquille au rayon du point chaud, énergie interne du DT) on été atteintes, et qu'un progrès décisif ne peut désormais être obtenu qu'au prix d'une augmentation de l'énergie des drivers.

L'ensemble de ces résultats a incité deux grands laboratoires, le CEA-Dam en France et le LLNL aux États-Unis, à s'engager dans la construction de deux lasers de grandes puissances, respectivement le LMJ (Laser Mégajoule) et le NIF (National lgnition Facility). Ces installations devraient permettre non seulement d'atteindre l'allumage, mais de réaliser la combustion du DT avec un gain élevé.

\section{Le projet Laser Mégajoule}

Le LMJ est conçu pour obtenir l'ignition et la combustion d'un mélange de deutérium-tritium avec un gain supérieur à un. Il a été calculé qu'une énergie de $\sim 2 \mathrm{MJ}$ en sortie du laser était nécessaire; pour l'obtenir avec une marge de sécurité raisonnable, 240 faisceaux laser ont été prévus. Le verre dopé au néodyme a été conservé comme milieu actif, pour ses qualités de stockage d'énergie et parce que sa technologie est bien développée, mais, afin de diminuer au maximum le coût de construction de l'installation, plusieurs innovations ont été étudiées et adoptées par rapport à la structure des précédents lasers, entre 
autres le regroupement des faisceaux laser en chaînes de huit faisceaux et la focalisation par réseaux.

Les 240 faisceaux du LMJ sont donc constitués en fait de 30 chaînes comportant chacune huit faisceaux. Ces 30 chaînes sont implantées dans deux halls laser situés de chaque coté de la salle d'expériences.

Il a été décidé de vérifier la validité de cette conception en grandeur réelle en construisant, en avance de phase, un prototype industriel de l'une des 30 chaînes du LMJ. Ce prototype est la ligne d'intégration laser (LIL) qui vient d'être qualifiée et dont l'un des faisceaux a prouvé l'obtention des performances requises.

La réalisation du laser LMJ, prévue pour 2011, est en bonne voie. Les concepts et la faisabilité des composants ayant été vérifiés avec succès sur la LIL, le choix des industriels a été effectué et les contrats correspondants ont été passés ; le montage de ces composants dans le bâtiment en cours de construction pourra commencer dès 2007.

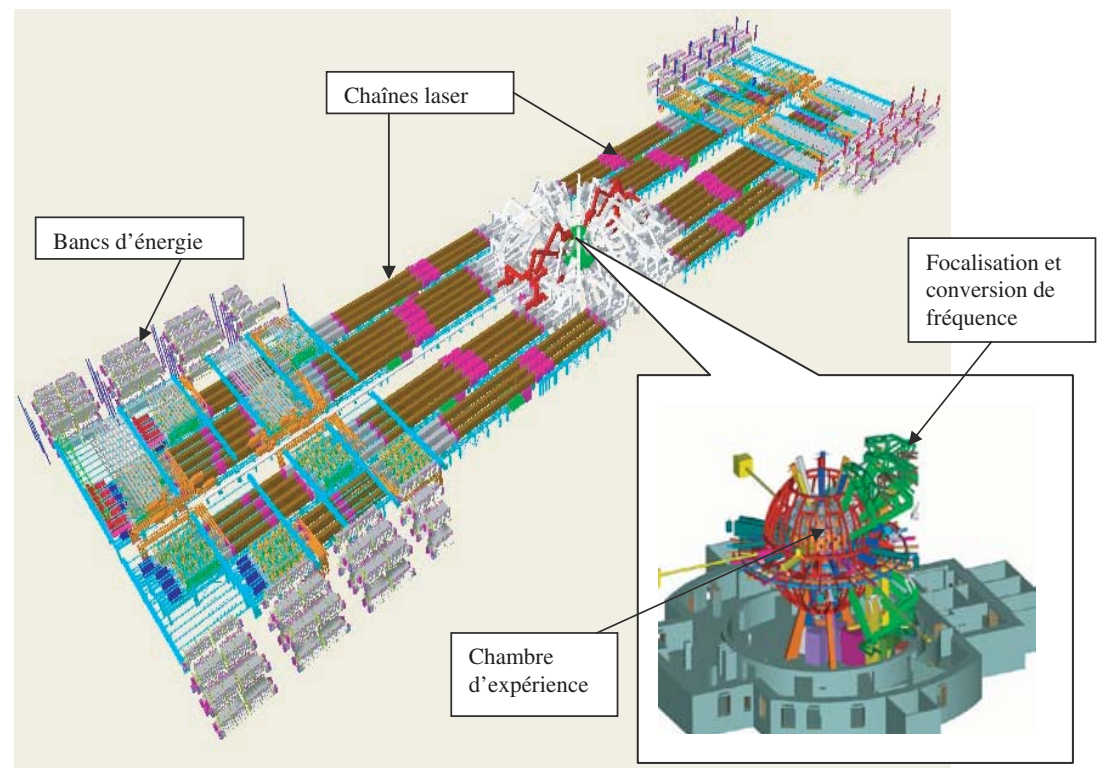

Figure 2.3

Architecture du Laser Mégajoule.

La collaboration entre la France et les États-Unis en matière de technologie des lasers de forte énergie remonte aux années 1970. L'idée de construire un laser de la classe mégajoule ayant été retenue en 1990, un accord officiel 
de collaboration fut signé en 1994 entre le CEA et le DOE (Departement of Energy) américain. Cet accord portait en particulier sur la mise au point des nouvelles technologies nécessaires et plus généralement sur la conception d'un tel laser. Diverses actions ont été menées en commun entre les deux laboratoires : conception et test $d^{\prime}$ 'amplificateurs à plaques, développement $d^{\prime}$ un procédé de fabrication de verre laser par coulée continue, développement d'un procédé de croissance cristalline permettant l'obtention de monocristaux de KDP, etc.

Depuis 1999, la construction des lasers NIF et LMJ se poursuit en parallèle. Les conceptions des deux lasers sont proches même si certains choix ont été différents, tels que le nombre de faisceaux nécessaires (240 pour LMJ contre 192 pour NIF) ou le principe de focalisation (par réseaux pour LMJ et par lentille pour NIF) par exemple.

Attaque directe vs indirecte

Chaque solution présente des avantages et des inconvénients :

- I'attaque directe est intéressante au plan du rendement de transfert énergétique entre le driver et le microballon, mais son point faible est l'uniformité d'irradiation (et par conséquent la stabilité de l'implosion) qui, pour être satisfaisante, requiert des aménagements optiques très sophistiqués ;

- I'attaque indirecte est ouverte à tous les types de drivers. Elle bénéficie de l'uniformité d'irradiation qu'apporte la structure de " corps noir » que constitue la cavité renfermant le microballon, mais pêche par un faible rendement énergétique.

Il en résulte que les grands projets - LMJ et NIF - ménagent la possibilité de basculer d'une configuration à l'autre selon les résultats.

\section{L'allumage latéral}

Le concept de l'allumage latéral est très attrayant et paraît prometteur; toutefois, il est hasardeux d'en vouloir préciser aujourd'hui le réalisme et l'intérêt vis-à-vis de l'allumage par point chaud central, par exemple en termes d'économie d'énergie du driver, car les mécanismes à mettre en œuvre sont encore trop méconnus pour être valablement quantifiés. Les travaux menés par l'ensemble de la communauté scientifique devraient clarifier la question dans la décennie à venir. 
Prospective laser

Malgré leur fragilité optique, I'utilisation des lasers pour un réacteur n'est pas exclue, à condition d'améliorer leurs performances. Pour aborder le problème $\mathrm{d}^{\prime}$ un driver laser de réacteur fonctionnant en cadence à quelques $\mathrm{Hz}$, on note que les laboratoires s'accordent pour explorer trois axes de recherche technologique : choix des matériaux (afin de gérer les effets thermiques), pompage par diode laser pour améliorer le rendement (mais le coût de ces diodes est encore prohibitif), définition d'une architecture permettant de garantir la qualité de la tache focale à obtenir sur la cible.

\subsection{L'implosion par ions lourds}

L'équivalent pour les ions de l'état des connaissances du domaine laser n'est pas acquis, les études actuelles portant sur le ralentissement des particules dans les plasmas ou la matière froide.

Les accélérateurs d'ions lourds actuels, tels que ceux du Cern par exemple, ont des caractéristiques intéressantes (cadence, stabilité, rendement), très supérieures à celles des lasers de puissance actuels, mais leur puissance instantanée est inférieure d'au moins trois ordres de grandeur à ce qui est nécessaire à un réacteur de fusion. Une question importante demeure : la focalisation. Au cours de l'accélération des particules, divers mécanismes peuvent induire des mouvements aléatoires des ions qui peuvent empêcher une focalisation suffisamment fine. La difficulté, si l'on veut progresser vers des études d'implosion significatives, est qu'il n'est pas possible, à la différence des lasers, de concevoir un prototype autrement qu'à l'échelle 1 pour obtenir la densité de flux d'énergie requise.

Les recherches au niveau « démonstration de principe " pour le driver (Proof Of Principle - POP - des Anglo-saxons) ont débuté aux États-Unis il y a quelques années, dans le cadre du VNL (Virtual National Laboratory), regroupant les trois grands programmes sur les accélérateurs (LBNL, LLNL et PPPL), et les participations de nombreuses institutions américaines. Au plan de l'irradiation d'une cible, un premier objectif serait de réaliser à court terme (5 ans) une expérience intégrée permettant de porter une cible dense à une température de $\sim 1600 \mathrm{~K}$ afin de réaliser des expériences de physique des hautes densités. L'étape suivante vise à gagner un facteur 10 sur la température. 


\subsection{L'implosion par Z-pinch}

Les résultats obtenus par les laboratoires de la Sandia (États-Unis) avec la "Z-Machine » montrent l'intérêt d'une telle installation comme driver : il a été fait état en 1996 d'une production de rayonnement $X$ très importante, et une émission neutronique d'origine thermonucléaire a été observée avec une capsule de renfermant du deutérium.

L'objectif de cette installation est maintenant de faire la démonstration d'un gain thermonucléaire élevé avec la possibilité d'un fonctionnement répétitif. Toutefois, I'application à des fins énergétiques reste à prouver.

\section{Conclusion}

\section{Démonstration de l'allumage et de la combustion du DT}

Les études longues et poussées réalisées par divers laboratoires ont conduit à cerner avec précision les structures de base des cibles à gain. Le concept (allumage et gain élevé) devrait être validé en laboratoire dans la prochaine décennie avec les deux installations laser LMJ (France) et NIF (États-Unis), qui devraient faire la démonstration de la première étape du développement de la fusion inertielle comme source d'énergie civile.

\section{Les projets de réacteurs}

Des projets nombreux et variés ont été publiés depuis les années 1970, ils sont américains pour la plus grande partie (la France ne dispose aujourd'hui $d^{\prime}$ 'aucune étude propre de réacteur inertiel). La variété résulte de la possibilité $d^{\prime}$ 'associer plusieurs types de chambres aux différents drivers envisagés.

La réalisation d'une chambre de réacteur est un problème complexe; la difficulté concerne à la fois le concept et les matériaux à mettre en jeu. Les recherches technologiques ont été faiblement développées sur ces sujets (bien que depuis quelques années des expériences de laboratoire soient menées sur la définition de matériaux et les simulations numériques; on peut associer cet intérêt renouvelé au développement des projets NIF et LMJ), et de nombreux challenges demeurent, essentiellement liés à la nature pulsée du dégagement d'énergie.

Certaines problématiques de la chambre de réaction sont similaires à celles du confinement magnétique : matériaux de couverture, récupération du tritium, 
extraction des matériaux activés, récupération de l'énergie dégagée. C'est à l'évidence un domaine où pourrait se développer une importante synergie entre les deux communautés de la fusion.

En termes de drivers, les lasers à verre sont aujourd'hui les plus avancés et leur utilisation pour un réacteur n'est pas écartée.

Néanmoins, il est généralement admis que lorsque les lasers auront démontré la possibilité de brûler du DT en laboratoire, les accélérateurs d'ions lourds pourraient être les drivers les mieux adaptés pour les applications énergétiques, en raison de leur rendement et de leur robustesse. L'échéance apparaît toutefois plus lointaine, car la réalisation d'un accélérateur d'ions lourds à l'échelle d'un réacteur représente un investissement et un saut technologique considérables qui ne pourra s'envisager que si le coût peut être réduit d'un ordre de grandeur par rapport à celui des technologies actuelles.

Les résultats obtenus par les laboratoires de la Sandia (États-Unis) avec la ZMachine (striction magnétique) montrent l'intérêt d'une telle installation comme driver, mais l'application à des fins énergétiques, principalement en termes de répétitivité, reste à prouver.

La fabrication des cibles reste également un des challenges de la fusion inertielle. Bien que l'implosion par laser soit la voie la plus avancée et que des techniques très sophistiquées de fabrication de cibles aient été mises au point pour les expériences d'interaction laser-matière, peu d'expériences mettant en jeu une cible similaire à celles conçues pour obtenir la combustion du DT (c'est-àdire possédant une couche cryogénique) ont été réalisées aujourd'hui. De plus, ces expériences sont réalisées avec une cadence de quelques tirs par jour. II est clair que, par rapport aux actuelles procédures de laboratoires, toutes les opérations de fabrication, de contrôle, de mise en place des cibles pour un réacteur travaillant à une fréquence de quelques hertz seront complètement à repenser, $d^{\prime}$ autant que l'objectif, en termes économiques est un coût unitaire ne dépassant pas $\sim 0,3 €$.

On peut enfin noter qu'une concertation au niveau européen entre des laboratoires intéressés par la fusion inertielle, rapprochant des compétences reconnues, variées et de haut niveau, profiterait au développement des réflexions sur les divers concepts de drivers et de chambres et permettrait de cerner les options à approfondir en vue de la conception d'un réacteur. 